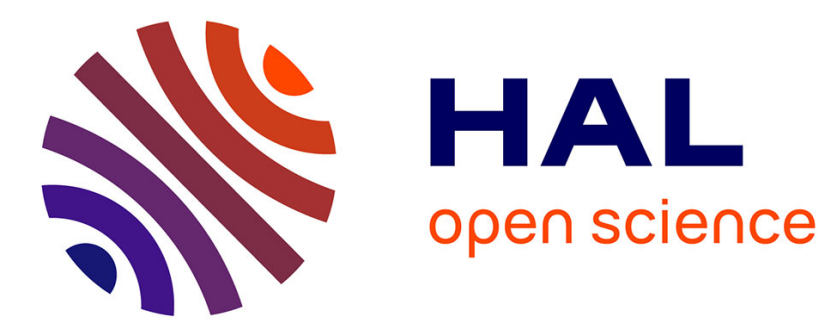

\title{
Plastoglobules: versatile lipoprotein particles in plastids
}

Claire Bréhélin, Felix Kessler, Klaas J van Wijk

\section{To cite this version:}

Claire Bréhélin, Felix Kessler, Klaas J van Wijk. Plastoglobules: versatile lipoprotein particles in plastids. Trends in Plant Science, 2007, 12 (6), pp.260-266. 10.1016/j.tplants.2007.04.003 . hal02106327

\section{HAL Id: hal-02106327 \\ https://hal.science/hal-02106327}

Submitted on 18 Jul 2019

HAL is a multi-disciplinary open access archive for the deposit and dissemination of scientific research documents, whether they are published or not. The documents may come from teaching and research institutions in France or abroad, or from public or private research centers.
L'archive ouverte pluridisciplinaire HAL, est destinée au dépôt et à la diffusion de documents scientifiques de niveau recherche, publiés ou non, émanant des établissements d'enseignement et de recherche français ou étrangers, des laboratoires publics ou privés. 


\title{
Plastoglobules: versatile lipoprotein particles in plastids
}

\section{Claire Bréhélin ${ }^{1}$, Felix Kessler ${ }^{1}$ and Klaas J. van Wijk ${ }^{2}$}

\author{
${ }^{1}$ Laboratoire de Physiologie Végétale, Institut de Biologie, Université de Neuchâtel, Emile Argand 11, CP 158, \\ CH-2009 Neuchâtel, Switzerland \\ ${ }^{2}$ Department of Plant Biology, Cornell University, Ithaca, NY 14853, USA
}

\begin{abstract}
Plastoglobules are plastid-localized lipoprotein particles that contain tocopherols and other lipid isoprenoidderived metabolites, as well as structural proteins named plastoglobulins. Surprisingly, recent publications show that plastoglobules contain enzymes involved in the metabolism of these secondary metabolites, as well as enzymes of unknown function. The size and number of plastoglobules vary during plastid development and differentiation, and strongly increase during light stress, senescence and in mutants blocked in thylakoid formation. Given that plastoglobules are contiguous with the outer lipid leaflet of the thylakoid membrane, it is highly plausible that a function of plastoglobules is the active channeling of lipid molecules and lipid breakdown products. Understanding the function of plastoglobules should provide a foundation for improving the nutritional value and yield of plants.
\end{abstract}

\section{History of plastoglobule research and discovery}

Early electron microscopic studies revealed the presence of 'osmiophilic globuli' inside chloroplasts (Figure 1) and chromoplasts, as well as other plastid types [1]. The diameter of these bodies, later termed plastoglobules, ranges from $30 \mathrm{~nm}$ to $5 \mu \mathrm{m}$. These plastoglobules could be conveniently isolated by flotation density centrifugation because of their relatively high lipid content $[1,2]$. The lipid composition of plastoglobules has been determined in several plant species - it consists mainly of prenyl-quinones and neutral lipids. Plastoglobules qualify as lipoprotein particles because they have been reported to associate with proteins. Members of the plastoglobulin family (also called fibrillin or PAP for plastid lipid-associated protein) [3], were the first known genuine plastoglobule protein components. In addition to vascular plants, plastoglobules are found in non-vascular species such as moss [4] and algae [5,6]. Interestingly, carotenoid-rich plastoglobule-like structures constitute the eyespot structure of Chlamydomonas reinhardtii, the proteome of which has been shown to contain members of the plastoglobulin family [6]. In cyanobacteria, the presence of 'lipid droplets' among the thylakoids has been reported [7]. The exact identity of these lipid droplets has not been defined; however, the presence of at least two plastoglobulin homologs in the genome of Synechocystis PCC6803 suggests that they could be plastoglobules.

Corresponding author: Bréhélin, C. (claire.brehelin@unine.ch).

Available online xxxxxx.
Although plastoglobules were largely viewed as passive lipid and carotenoid storage particles, their varying size in different species, plastid types and developmental stages suggested a more dynamic role. Moreover, correlative evidence suggested that plastoglobules are involved in thylakoid development as well as disassembly: (i) etioplasts with poorly developed thylakoids have more plastoglobules than are found in chloroplasts, but the plastoglobule abundance decreased during thylakoid biogenesis [8-10]; (ii) in senescent chloroplasts, during thylakoid disassembly, plastoglobules enlarge and accumulate $[9,11,12]$; (iii) several thylakoid biogenesis mutants showed increased accumulation of plastoglobules (e.g. Refs [13,14]); (iv) plastoglobules have been shown to play a role in chloroplast to chromoplast transition and the formation of the colored carotenoid fibrils [15]. Indeed, fibrils are fibrillar structures that originate from plastoglobules during chloroplast to chromoplast transition [16]. Currently, a rapidly growing body of evidence suggests an active role for plastoglobules in metabolic and stress-response pathways, which all suggest that plastoglobules are a metabolic intersection between different plastid compartments.

\section{Plastoglobule composition}

Plastoglobules isolated from chloroplasts are known to contain the prenyl quinones, including plastoquinone and phylloquinone and $\alpha$-tocopherol $[1,2,9,17]$. Data from a recent study have shown that a significant fraction of phylloquinone (vitamin K1) in chloroplasts is not associated with photosystem I, but locates to plastoglobules [18]. This suggests that plastoglobules are a sink for the deposit of excess phylloquinone and its precursors. Whereas galactolipids have been detected in plastoglobules $[1,2,9]$, other studies have reported that galactolipids were absent, suggesting that they originated from contaminating thylakoid membranes $[17,19]$. Plastoglobules from chromoplasts also contain triacylglycerols, $\beta$-carotene and carotenoid esters $[2,17]$. The accumulation of carotenoids in the hydrophobic core of chromoplast plastoglobules and fibrils [16] confers color to fruits and petals. Fatty acid phytyl esters (FAPEs) have been shown to localize to plastoglobules and thylakoids [20]. Under nitrogen starvation, destabilizing compounds, such as acyl groups from membrane lipids and phytols from chlorophyll degradation, are released. Their incorporation into FAPEs and storage in plastoglobules probably prevents them from damaging plastid membranes. 


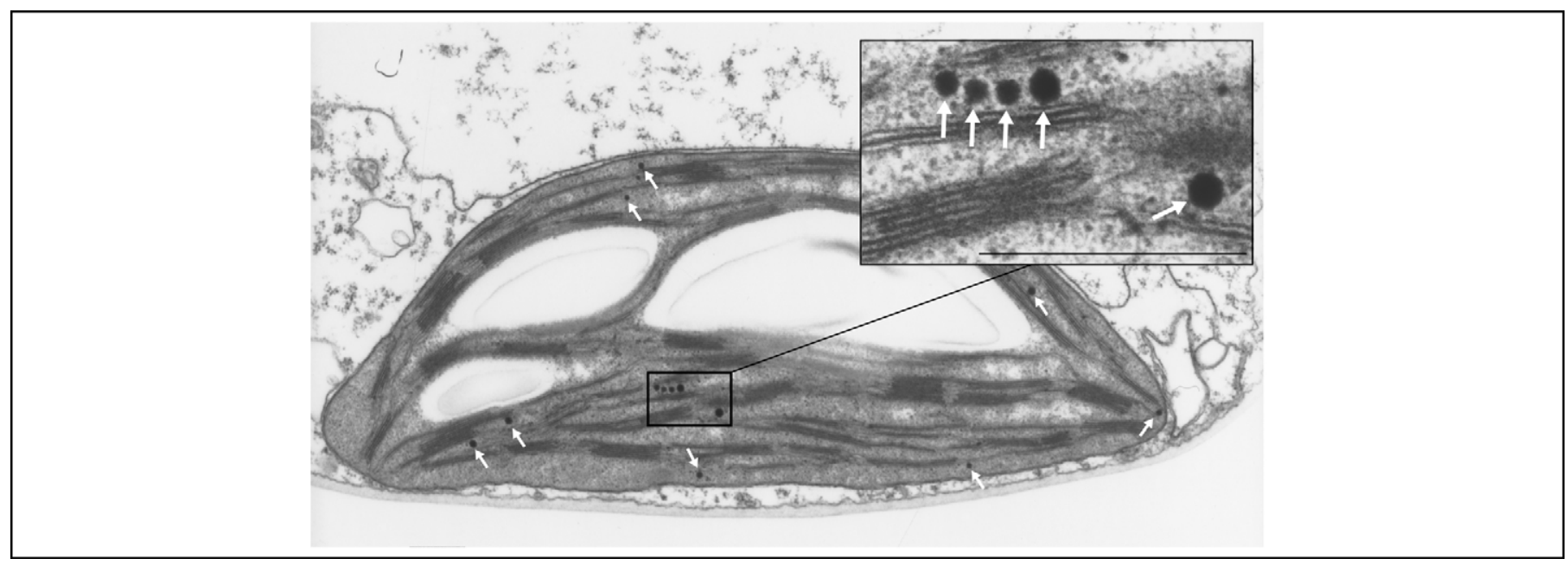

Figure 1. On electron micrographs of chloroplasts (here a one month-old Arabidopsis leaf), plastoglobules (indicated by white arrows) appear as small black globules in close proximity with thylakoids. Scale bar $=0.5 \mu \mathrm{m}$.

Plastoglobules also serve as lipid deposits in elaioplasts, notably in tapetum cells were these lipids are released and deposited on maturing pollen [21]. Immunogold-electron microscopy studies have shown that plastoglobulin proteins localize at the periphery of plastoglobules [22,23]. Fibrillin, the plastoglobulin protein present in chromoplasts received its name from its association with carotenoid fibrils [16].

\section{Plastoglobule proteome}

The advancement of proteomics and biological mass spectrometry greatly helped in the identification of plastoglobule proteins and functions. Thirty-four proteins are considered candidates for genuine plastoglobule proteins based on their experimental identification in purified plastoglobules isolated from Arabidopsis thaliana chloroplasts $[24,25]$. Twenty-three of the proteins were found in both of the two independent studies. The identified proteins fall into three categories: plastoglobulins/PAP/fibrillins, chloroplast and chromoplast metabolic proteins and unclassified proteins (Table 1).

\section{Plastoglobulins}

The plastoglobulin/PAP/fibrillin family in Arabidopsis consists of thirteen genes [3]. Eight members of this family were identified in the plastoglobule proteome, suggesting that the majority of the plastoglobulins/PAPs/fibrillins function in plastoglobules. Moreover, expression of several of the identified plastoglobulins fused to GFP gave punctuate fluorescence patterns consistent with plastoglobule localization [24]. Several names have been used to describe these proteins and, for clarity, we propose the name 'plastoglobulin' for future reference of this family. The name 'fibrillin' to describe these proteins appears somewhat limited because it suggests a restricted localization in carotenoid-rich fibrils in chromoplasts, whereas both FIB (as an abbreviation for fibrillin) and PAP for 'plastid lipid associated protein' have already been defined by The International Arabidopsis Community (TAIR; http:// www.Arabidopsis.org), as phosphatidic acid phosphatase and fibrillarin, respectively. Thus, we propose to use the term plastoglobulin (abbreviated to PGL) to name members of this family.
Several of the plastoglobulins were previously identified in the thylakoid proteome $[26,27]$, and have later been attributed to plastoglobules co-isolated together with thylakoids [24,25]. Homologs of these plastoglobulins and proteins of unknown function have also been identified in plastoglobules isolated from pepper chromoplasts [25], suggesting a functional and structural relationship between plastoglobules from chloroplasts and chromoplasts. Although no enzymatic activity has been reported for any plastoglobulin, the red pepper plastoglobulin has been shown to mediate in vitro fibril assembly [16], and overexpression of tobacco plastoglobulin increased plastoglobule number in vivo [28], suggesting that the PGLs have a predominantly structural role, possibly regulating size and shape of lipoprotein structures in plastids. Many questions regarding the role of plastoglobulins remain. For example: (i) what is their role in plastoglobule formation? (ii) Are they specific to different kinds of plastoglobules within the same plastid type or belonging to a specific type of plastid? (iii) Do they all function in plastoglobules or is a subset associated with the thylakoid or other plastid membranes?

\section{Chloroplast metabolic enzymes}

Although the presence of plastoglobulins was not unexpected, the identification of known metabolic enzymes was surprising given that plastoglobules were generally considered passive lipid storage bodies [9,19]. Three chloroplast enzymes involved in biosynthetic pathways related to stress responses were identified: the allene oxide synthase implicated in jasmonate synthesis, a 9-cis-epoxycarotenoid dioxygenase that might participate in carotenoid and ABA metabolism, and tocopherol cyclase (VTE1, vitamin E deficient 1), a key enzyme in vitamin $\mathrm{E}$ synthesis. In addition, the plastoglobule proteome also contained three fructose-bisphosphate aldolases that participate in the Calvin cycle and glycolysis, one of which appears to be specific for plastoglobules [25]. Transient expression of fructosebisphosphate aldolases fused to GFP in protoplasts confirmed their presence in plastoglobules [24], but most of the aldolase activity was found in the stroma. However, aldolase assays showed high specific activity in plastoglobules, amounting to $\sim 10 \%$ of total activity in chloroplasts 
Table 1. Thirty-four proteins identified by tandem mass spectrometry in plastoglobules isolated from Arabidopsis thaliana chloroplasts

\begin{tabular}{|c|c|c|c|}
\hline Name & AGI accession number & Function or TAIR description & Refs $^{a}$ \\
\hline AtPGL30.4/FIB4 & At3g23400 & PGs protein coat & {$[24,25]$} \\
\hline AtPGL33/FIB1b & At4g22240 & PGs protein coat & {$[24,25]$} \\
\hline AtPGL35/FIB1a & At4g04020 & PGs protein coat & {$[24,25]$} \\
\hline AtPGL40/FIB2 & At2g35490 & PGs protein coat & {$[24,25]$} \\
\hline AtPGL25/FIB3a & At3g26070 & PGs protein coat & [24] \\
\hline FBA1 & At2g21330 & Fructose-bisphosphate aldolase & {$[24,25]$} \\
\hline Putative FBA & At2g01140 & Fructose-bisphosphate aldolase & {$[24,25]$} \\
\hline FBA2 & At4g38970 & Fructose-bisphosphate aldolase & {$[24,25]$} \\
\hline AOS allene oxide synthase & At5g42650 & Jasmonate synthesis & {$[24,25]$} \\
\hline NCED4/CDD4 & At4g19170 & Neoxanthin cleavage enzyme & {$[24,25]$} \\
\hline VTE1 tocopherol cyclase & At4g32770 & Vitamin E synthesis & {$[24,25]$} \\
\hline Unknown & At1g78140 & UbiE methyltransferase-related & {$[24,25]$} \\
\hline Unknown & At2g41040 & UbiE methyltransferase-related & {$[24,25]$} \\
\hline Unknown & At1g54570 & Esterase/lipase/thioesterase family & {$[24,25]$} \\
\hline Unknown & At3g26840 & Esterase/lipase/thioesterase family & {$[24,25]$} \\
\hline Unknown & At4g13200 & Expressed protein & {$[24,25]$} \\
\hline Unknown & At5g05200 & ABC1 kinase family & {$[24,25]$} \\
\hline Unknown & At1g79600 & $A B C 1$ kinase family & {$[24,25]$} \\
\hline Unknown & At4g31390 & $A B C 1$ kinase family & {$[25]$} \\
\hline Unknown & At1g71810 & $\mathrm{ABC} 1$ kinase family & {$[25]$} \\
\hline Unknown & At1g09340 & Expressed protein (Rap38) & {$[25]$} \\
\hline Unknown & At3g63140 & mRNA binding protein (Rap41) & [25] \\
\hline Unknown & At1g52590 & Expressed protein & [24] \\
\hline Unknown & At1g26090 & Expressed protein & [25] \\
\hline
\end{tabular}

Abbreviation: PG, plastoglobule.

aProteins were identified in either Ref. [24] or [25] or in both.

(C. Bréhélin and F. Kessler, unpublished). It has been proposed that this dual localization of fructose-bisphosphate aldolases might have a regulatory role in their enzymatic activity. Similarly, the role of most metabolic enzymes in plastoglobules is not yet fully understood, but inroads have been made in the case of VTE1.

VTE1 catalyzes the conversion of 2,3-dimethyl-5-phytyl1,4-hydroquinol (DMPQ) to $\gamma$-tocopherol [29]. Immunoelectron microscopy and transient expression of a GFP-fusion protein substantiated the specific association of VTE1 with plastoglobules [24,30]. In addition, it has been shown that tocopherols are highly enriched in Arabidopsis plastoglobules, suggesting that plastoglobules are an important site of tocopherol synthesis and accumulation. However, the known enzyme activities in tocopherol biosynthesis VTE2 or HPT1 (homogentisate phytyltransferase), VTE3 (2methyl-6-phytyl-1,4-hydroquinol methyltransferase) and VTE4 ( $\gamma$-tocopherol methyltransferase) [31], with the possible exception of VTE1, have all been localized to the inner envelope membrane [32]. But at the protein level, only VTE3 (also called APGs1 or E37) has been demonstrated to be present at the inner envelope [3335], a finding confirmed by large-scale proteome studies of the chloroplast envelope $[36,37]$. To the best of our knowledge, there is no prior experimental evidence for the protein localization of VTE1 and VTE4, the enzymes functioning downstream of VTE3. Thus, the consistent identification of VTE1 in plastoglobule preparations from chloroplasts and chromoplasts $[24,25]$ makes a strong case for its localization in plastoglobules; the location of VTE4 remains to be determined. We note that VTE1 and VTE4 have no predicted transmembrane domains, whereas VTE2 and VTE3 have nine and one predicted transmembrane domains, respectively, compatible with their insertion into a lipid bilayer such as the inner envelope. It is possible that VTE4 is peripheral to both plastoglobules and inner envelopes. The findings suggest that plastoglobules are not only involved in synthesis and storage of tocopherol, but also directly or indirectly involved in the trafficking of tocopherol and its precursors between the inner chloroplast membrane and the thylakoids (Figure 2).

A carotenoid cleavage dioxygenase, CCD4 or NCED4 [38], has been identified in plastoglobules of chloroplasts, but its substrates and cleavage products are not known. Stable isotope experiments have indicated a doubling of NCED4 accumulation in plastoglobules after dark treatment compared with high light treatment, suggesting an active role in dark-induced breakdown of carotenoids [25]. 


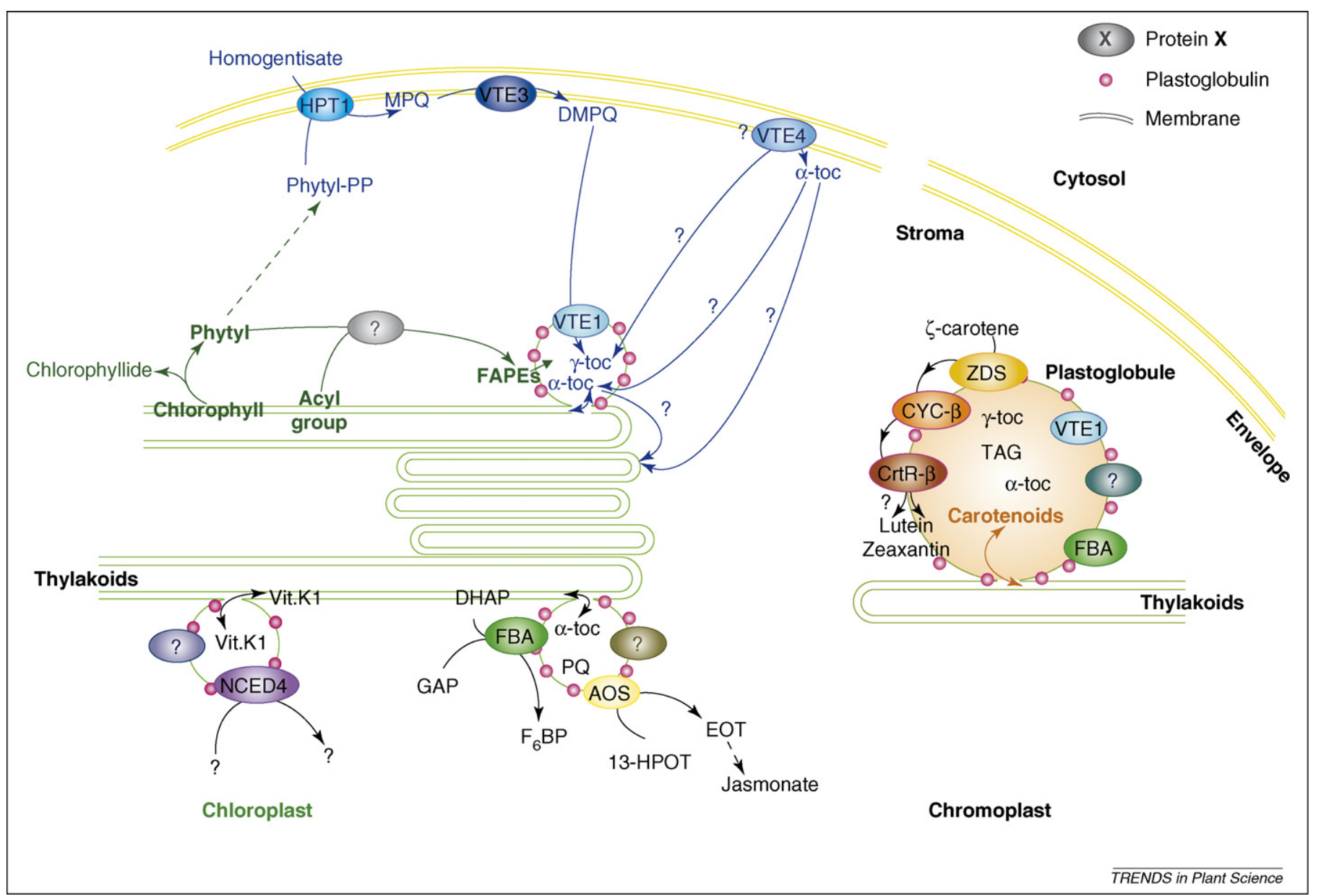

Figure 2. Plastoglobules are involved in diverse metabolic pathways in chloroplasts and chromoplasts. Chloroplast and chromoplast membrane systems are depicted. Plastoglobules are attached to thylakoids via the outer leaflet of the lipid bilayer. Plastoglobules in chromoplasts are typically larger than in chloroplasts and accumulate colored carotenoids. Enzymes from diverse pathways that might involve plastoglobules are represented by colored ellipses. The first steps of the tocopherol biosynthesis (depicted in blue) are located at the envelope, where HPT1 (homogentisate phytyltransferase) produces MPQ (2-methyl-6-phytyl-1,4-hydroquinol). MPO is converted into DMPO (2,3-dimethyl-5-phytyl-1,4-hydroquinol) by VTE3. DMPO is the substrate of the plastoglobule enzyme VTE1, which produces $\gamma$-toc ( $\gamma$-tocopherol), later converted in $\alpha$ toc ( $\alpha$-tocopherol) by VTE4 [31]. VTE4 localization is uncertain, in this review it is suggested to be at the periphery of the envelope or the plastoglobules. Free phytol, a breakdown product of chlorophyll degradation, is bound to acyl groups by an unknown enzyme (gray ellipse with question mark) to produce fatty acid phytyl esters (FAPEs), which are stored in plastoglobules. AOS (allene oxide synthase), involved in jasmonate biosynthesis, converts 13-HPOT (13-hydroperoxylinolenic acid) to an unstable EOT (12,13-epoxyoctadecatrienoic acid) [61]. FBA (fructose-1,6-bisphosphate aldolase) converts DHAP (dihydroxyacetone-phosphate) and GAP (glyceraldehyde-3phosphate) in $\mathrm{F}_{6} \mathrm{BP}$ (fructose-1,6-bisphosphate). Substrates and products of NCED4 (carotenoid dioxygenase enzyme) are unknown [38]. Plastoglobules have been shown to contain plastoquinone (PQ) in addition to tocopherols. Phylloquinone (Vit.K1) is distributed between thylakoids and plastoglobules. The continuity of plastoglobules with the outer leaflet of thylakoids would enable the channeling (symbolized as a double-headed arrow) of diverse metabolites such as phylloquinone and tocopherols. Plastoglobules from chromoplasts also contain carotenoids and TAG (triacyl glycerol). ZDS ( $\zeta$-carotene desaturase), CYC- $\beta$ (lycopene- $\beta$-cyclase) and CrtR- $\beta$ ( $\beta$-carotene- $\beta$ -

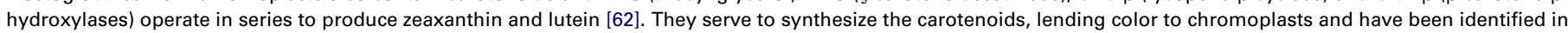
plastoglobules from chromoplasts [25]. In addition, several proteins with unknown functions (ellipse with question mark) have been identified in plastoglobules.

\section{Metabolic enzymes in plastoglobules of chromoplasts in red pepper}

Chromoplasts of ripe red peppers do not contain thylakoid membranes or chlorophylls, but instead accumulate large amounts of carotenoids that are mostly sequestered in fibrillar plastoglobules [16]. Proteome analysis of isolated plastoglobules from the chromoplasts of ripe red peppers identified $\zeta$-carotene desaturase, lycopene $\beta$-cyclase, and two $\beta$-carotene $\beta$-hydroxylases operating in series in bicyclic carotenoid biosynthesis [25]. This suggests that plastoglobules in chromoplasts have a specific enzymatic function in carotenoid biosynthesis (Figure 2), in addition to their well known function of carotenoid storage and sequestration [15-17].

\section{Unclassified proteins in plastoglobules}

Among the 20 unclassified plastoglobule proteins, six are putatively involved in quinone synthesis and two in general lipid metabolism on the basis of predicted functional domains (Table 1). The four ABC1 kinases might function in regulation of quinone synthesis, based on the role of their homologs in Escherichia coli and Saccharomyces cerevisiae (see Ref. [25] for discussion). Their substrates and products are currently not known. The key to understanding their enzymatic function could be the determination of the plastoglobule metabolome, in combination with detailed enzyme activity measurements. It could then be possible to correlate quinolic or lipidic substrates or products contained in plastoglobules with these unknown plastoglobule proteins.

\section{Ultrastructure of plastoglobules}

Given that plastoglobules participate in various metabolic pathways as well as in lipid storage, the question arises as to how these lipids can be transferred to and from the thylakoid membranes. Plastoglobules often appear in close proximity to thylakoid membranes and physical connections have 
been reported. Electron tomography has shown that virtually all plastoglobules are attached to thylakoids, some of them directly and others via a network of interconnected plastoglobules [30]. Moreover, it has been shown that the outer lipid leaflet of the thylakoid membrane is in direct continuity with the polar lipid layer surrounding the plastoglobules [30] (Figure 2). This arrangement might provide the means for a direct lipid conduit for 'metabolite channeling' between the thylakoid membrane and the plastoglobules. In addition, the tomography analyses demonstrate that plastoglobulin AtPGL35 (At4g04020) 'coats' the plastoglobules, whereas the tocopherol cyclase penetrates the polar lipid monolayer, potentially giving access to neutral lipid substrates in the plastoglobule interior [30].

\section{Involvement of plastoglobules in stress response}

The first indications that plastoglobules are involved in stress responses came from ultra-structural observations. Indeed, several studies have reported the presence of larger and more numerous plastoglobules in chloroplasts from plants grown under diverse stress conditions [39-47]. In addition, the expression of $P G L$ genes has been shown to be modulated by diverse stress stimuli such as exposure to reactive oxygen species [48,49] or ozone [50], ABA induction [51,52], wounding [3,48,50,53], bacterial infection [49] and a variety of other environmental stress conditions [3,48,50,51,53-55].

Systematic and quantitative comparative proteomics have shown that the predominant change in the Arabidopsis peripheral thylakoid proteome upon light stress treatment is the several-fold up-regulation of plastoglobule associated proteins [56]. Arabidopsis and tobacco plants with increased levels of plastoglobulin transcripts show enhanced tolerance to light stress $[28,52]$. By contrast, plants with reduced levels of plastoglobulin AtPGL35 show more pronounced inhibition of photosystem II under light stress. ABA can also regulate the response of plastoglobules to environmental stress. Indeed, the expression and accumulation of plastoglobulin AtPGL35 is regulated by $\mathrm{ABA}$ response regulators $\mathrm{ABI} 1$ and $\mathrm{ABI} 2$ [30,51,52].

The mechanism, and the role of this modulation of plastoglobule size and number in relation to stress are not yet understood. We hypothesize that the enlargement of plastoglobules correlates with high levels of plastoglobulin proteins and the increased production of small (antioxidant) molecules such as tocopherols. As discussed earlier, plastoglobules are directly implicated in the synthesis and storage of the antioxidant tocopherols [24]. Moreover, tocopherols have been proposed to protect membrane lipids from photooxidation and to protect photosystem II from photoinactivation [57]. Thus, under oxidative conditions, the tocopherols stored in plastoglobules would be delivered to thylakoid membranes to scavenge reactive oxygen species (Figure 2). This delivery ('metabolite channeling') would be possible via the structural connection between plastoglobules and thylakoids as described in Ref. [30]. However, a recent study [58] demonstrates that tocopherols not only have a role in photoprotection but are also necessary for adaptation to low temperatures and phloem loading. In light of this result, it will be interesting to study the role of plastoglobules in low temperature adaptation.

\section{Integration of plastoglobule functions with chloroplast metabolism and stress responses}

The recent publications have laid a foundation for the molecular understanding of plastoglobule functions in chloroplast and chromoplast secondary metabolism and stress responses. These discoveries strongly suggest that plastoglobules actively participate in diverse secondary metabolism pathways and stress responses and, thus, are not merely a 'passive storage' compartment but rather versatile particles. However, the plastoglobule-localized metabolic activities are part of larger networks of metabolic reactions and pathways located, in part, in other compartments of the chloroplast. This implies trafficking of substrates and products to and from the plastoglobules. Future research must address how plastoglobules participate in such intra-chloroplast metabolite trafficking. Metabolite analysis using modern mass spectrometry-based techniques [59] will probably lead to the identification of new plastoglobule metabolites. In combination with reverse genetic techniques, this might enable the functions of plastoglobule proteins of presently unknown enzymatic function to be unraveled. The role of the plastoglobulin family in the formation of plastoglobules in the various plastid types and their role in the dynamic response to various stress and developmental conditions remains to be determined. Finally, plastoglobules have interesting potential for use in applied biotechnology or molecular farming, such as the production of recombinant proteins in plants [60] and increased accumulation of lipophilic vitamins.

\section{Acknowledgements}

We thank Michèle Vlimant (Laboratory of Animal Physiology, Neuchâtel) for her skilled help in electron microscopy. Funding was provided to K.J.V.W. by the US Department of Agriculture (USDA-Plants and Environment \#0195698). C.B. and F.K. thank the Swiss National Center for Competence in Research (NCCR Plant Survival) and the University of Neuchâtel for financial support.

\section{References}

1 Greenwood, A.D. et al. (1963) The osmiophilic globules of chloroplasts. I. Osmiophilic globules as a normal component of chloroplasts and their isolation and composition in Vicia faba L. Biochim. Biophys. Acta 78, 148-162

2 Bailey, J.L. and Whyborn, A.G. (1963) The osmiophilic globules of chloroplasts. II. Globules of the spinach-beet chloroplast. Biochim. Biophys. Acta 78, 163-174

3 Laizet, Y. et al. (2004) Subfamily organization and phylogenetic origin of genes encoding plastid lipid-associated proteins of the fibrillin type. J. Genome Sci. Tech. 3, 19-28

4 Rinnan, R. and Holopainen, T. (2004) Ozone effects on the ultrastructure of peatland plants: Sphagnum mosses, Vaccinium oxycoccus, Andromeda polifolia and Eriophorum vaginatum. Ann. Bot. (Lond.) 94, 623-634

5 Katz, A. et al. (1995) Isolation and characterization of a protein associated with carotene globules in the alga Dunaliella bardawil. Plant Physiol. 108, 1657-1664

6 Schmidt, M. et al. (2006) Proteomic analysis of the eyespot of Chlamydomonas reinhardtii provides novel insights into its components and tactic movements. Plant Cell 18, 1908-1930

7 Stanier, G. (1988) Fine structure of cyanobacteria. In Methods in Enzymology (Packer, L. and Glazer, A.N., eds), pp. 157-172, Academic Press

8 Lichtenthaler, H.K. and Peveling, E. (1967) Plastoglobuli in Verschiedenen Differenzierungsstadien der Plastiden bei Allium cepa L. Planta (Berl.) 72, 1-13

9 Lichtenthaler, H.K. (1968) Plastoglobuli and the fine structure of plastids. Endeavor 27, 144-149 
10 Sprey, B. and Lichtenthaler, H. (1966) Zur Frage der Beziehungen zwischen Plastoglobuli und Thylakoidgenese in Gerstenkeimlingen. Z. Naturforschg 21b, 697-699

11 Guiamét, J.J. et al. (1999) Mass exodus from senescing soybean chloroplasts. Plant Cell Physiol. 40, 986-992

12 Ghosh, S. et al. (2001) Ultrastructural and biochemical changes in chloroplasts during Brassica napus senescence. Plant Physiol. Biochem. 39, 777-784

13 Rudella, A. et al. (2006) Downregulation of ClpR2 leads to reduced accumulation of the ClpPRS protease complex and defects in chloroplast biogenesis in Arabidopsis. Plant Cell 18, 1704-1721

14 Kroll, D. et al. (2001) VIPP1, a nuclear gene of Arabidopsis thaliana essential for thylakoid membrane formation. Proc. Natl. Acad. Sci. U. S. A. $98,4238-4242$

15 Vishnevetsky, M. et al. (1999) Carotenoid sequestration in plants: the role of carotenoid-associated proteins. Trends Plant Sci. 4, 232-235

16 Deruere, J. et al. (1994) Fibril assembly and carotenoid overaccumulation in chromoplasts: a model for supramolecular lipoprotein structures. Plant Cell 6, 119-133

17 Steinmüller, D. and Tevini, M. (1985) Composition and function of plastoglobuli. I. Isolation and purification from chloroplasts and chromoplasts. Planta 163, 201-207

18 Lohmann, A. et al. (2006) Deficiency in phylloquinone (vitamin $\mathrm{K}_{1}$ ) methylation affects prenyl quinone distribution, photosystem I abundance, and anthocyanin accumulation in the Arabidopsis AtmenG mutant. J. Biol. Chem. 281, 40461-40472

19 Tevini, M. and Steinmüller, D. (1985) Composition and function of plastoglobuli. II. Lipid composition of leaves and plastoglobuli during beech leaf senescence. Planta 163, 91-96

20 Gaude, N. et al. (2007) Nitrogen deficiency in Arabidopsis affects galactolipid composition and gene expression and results in accumulation of fatty acid phytyl esters. Plant J. 49, 729-739

$21 \mathrm{Wu}$, S.S. et al. (1997) Isolation and characterization of neutral-lipidcontaining organelles and globuli-filled plastids from Brassica napus tapetum. Proc. Natl. Acad. Sci. U. S. A. 94, 12711-12716

22 Pozueta-Romero, J.et al. (1997) A ubiquitous plant housekeeping gene, PAP, encodes a major protein component of bell pepper chromoplasts. Plant Physiol. 115, 1185-1194

23 Kessler, F. et al. (1999) Identification of proteins associated with plastoglobules isolated from pea (Pisum sativum L.) chloroplasts. Planta 208, 107-113

24 Vidi, P.A. et al. (2006) Tocopherol cyclase (VTE1) localization and vitamin $\mathrm{E}$ accumulation in chloroplast plastoglobule lipoprotein particles. J. Biol. Chem. 281, 11225-11234

25 Ytterberg, A.J. et al. (2006) Protein profiling of plastoglobules in chloroplasts and chromoplasts. A surprising site for differential accumulation of metabolic enzymes. Plant Physiol. 140, 984-997

26 Friso, G. et al. (2004) In-depth analysis of the thylakoid membrane proteome of Arabidopsis thaliana chloroplasts: new proteins, new functions, and a plastid proteome database. Plant Cell 16, 478-499

27 Peltier, J.B. et al. (2004) New functions of the thylakoid membrane proteome of Arabidopsis thaliana revealed by a simple, fast, and versatile fractionation strategy. J. Biol. Chem. 279, 49367-49383

28 Rey, P. et al. (2000) Over-expression of a pepper plastid lipid-associated protein in tobacco leads to changes in plastid ultrastructure and plant development upon stress. Plant J. 21, 483-494

29 Porfirova, S. et al. (2002) Isolation of an Arabidopsis mutant lacking vitamin $\mathrm{E}$ and identification of a cyclase essential for all tocopherol biosynthesis. Proc. Natl. Acad. Sci. U. S. A. 99, 12495-12500

30 Austin, J.R. et al. (2006) Plastoglobules are lipoprotein subcompartments of the chloroplast that are permanently coupled to thylakoid membranes and contain biosynthetic enzymes. Plant Cell $18,1693-1703$

31 DellaPenna, D. (2005) A decade of progress in understanding vitamin $\mathrm{E}$ synthesis in plants. J. Plant Physiol. 162, 729-737

32 Soll, J. et al. (1985) Localization of synthesis of prenylquinones in isolated outer and inner envelope membranes from spinach chloroplasts. Arch. Biochem. Biophys. 238, 290-299

33 Brink, S. et al. (1995) Sorting of nuclear-encoded chloroplast membrane proteins to the envelope and the thylakoid membrane. J. Biol. Chem. 270, 20808-20815

34 Dreses-Werringloer, U. et al. (1991) cDNA sequence and deduced amino acid sequence of the precursor of the $37-\mathrm{kDa}$ inner envelope membrane polypeptide from spinach chloroplasts. Its transit peptide contains an amphiphilic alpha-helix as the only detectable structural element. Eur. J. Biochem. 195, 361-368

35 Teyssier, E. et al. (1996) Is E37, a major polypeptide of the inner membrane from plastid envelope, an $S$-adenosyl methioninedependent methyltransferase? Plant J. 10, 903-912

36 Ferro, M. et al. (2003) Proteomics of the chloroplast envelope membranes from Arabidopsis thaliana. Mol. Cell. Proteomics 2, 325345

37 Froehlich, J.E. et al. (2003) Proteomic study of the Arabidopsis thaliana chloroplastic envelope membrane utilizing alternatives to traditional two-dimensional electrophoresis. J. Proteome Res. 2, 413425

38 Tan, B.C. et al. (2003) Molecular characterization of the Arabidopsis 9-cis epoxycarotenoid dioxygenase gene family. Plant J. 35, 4456

39 Locy, R.D. et al. (1996) Photosynthesis in salt-adapted heterotrophic tobacco cells and regenerated plants. Plant Physiol. 110, 321-328

40 Eymery, F. and Rey, P. (1999) Immunocytolocalization of CDSP 32 and CDSP 34, two chloroplastic drought-induced stress proteins in Solanum tuberosum plants. Plant Physiol. Biochem. 37, 305-312

41 Sallas, L. et al. (2003) Contrasting effects of elevated carbon dioxide concentration and temperature on Rubisco activity, chlorophyll fluorescence, needle ultrastructure and secondary metabolites in conifer seedlings. Tree Physiol. 23, 97-108

42 Utriainen, J. and Holopainen, T. (2001) Influence of nitrogen and phosphorous availability and ozone stress on Norway spruce seedlings. Tree Physiol. 21, 447-456

43 Utriainen, J. and Holopainen, T. (2001) Nitrogen availability modifies the ozone responses of Scots pine seedlings exposed in an open-field system. Tree Physiol. 21, 1205-1213

44 Paakkonen, E. et al. (1997) Differences in growth, leaf senescence and injury, and stomatal density in birch (Betula pendula Roth.) in relation to ambient levels of ozone in Finland. Environ. Pollut. 96, 117-127

45 Duret, S. et al. (1986) Cadmium-induced ultrastructural changes in Euglena cells. Environ. Res. 39, 96-103

46 Orsenigo, M. and Rascio, N. (1976) Chloroplast fine structure in the japonica-2 maize mutant exposed to continuous illumination. 1 . The green tissues. Cytobios 16, 171-182

47 Bondada, B.R. and Syvertsen, J.P. (2003) Leaf chlorophyll, net gas exchange and chloroplast ultrastructure in citrus leaves of different nitrogen status. Tree Physiol. 23, 553-559

48 Manac'h, N. and Kuntz, M. (1999) Stress induction of a nuclear gene encoding for a plastid protein is mediated by photo-oxidative events. Plant Physiol. Biochem. 37, 859-868

49 Langenkamper, G. et al. (2001) Accumulation of plastid lipidassociated proteins (fibrillin/CDSP34) upon oxidative stress, ageing and biotic stress in Solanaceae and in response to drought in other species. J. Exp. Bot. 52, 1545-1554

$50 \mathrm{Kim}, \mathrm{H} . \mathrm{U}$. et al. (2001) Brassica rapa has three genes that encode proteins associated with different neutral lipids in plastids of specific tissues. Plant Physiol. 126, 330-341

51 Gillet, B. et al. (1998) Molecular characterization of CDSP 34, a chloroplastic protein induced by water deficit in Solanum tuberosum L. plants, and regulation of CDSP 34 expression by ABA and high illumination. Plant J. 16, 257-262

52 Yang, Y. et al. (2006) Fibrillin expression is regulated by abscisic acid response regulators and is involved in abscisic acid-mediated photoprotection. Proc. Natl. Acad. Sci. U. S. A. 103, 6061-6066

53 Chen, H-C. et al. (1998) Drought- and wound-induced expression in leaves of a gene encoding a chromoplast carotenoid-associated protein. Plant J. 14, 317-326

54 Pruvot, G. et al. (1996) Characterization of a novel drought-induced $34-\mathrm{kDa}$ protein located in the thylakoids of Solanum tuberosum L. plants. Planta 198, 471-479

55 Pruvot, G. et al. (1996) Effects of low temperature, high salinity and exogenous $\mathrm{ABA}$ on the synthesis of two chloroplastic droughtinduced proteins in Solanum tuberosum. Physiol. Plant. 97, 123131

56 Giacomelli, L. et al. (2006) High light response of the thylakoid proteome in Arabidopsis wild type and the ascorbate-deficient mutant vtc2-2. A comparative proteomics study. Plant Physiol. 141, 685-701 
57 Havaux, M. et al. (2005) Vitamin E protects against photoinhibition and photooxidative stress in Arabidopsis thaliana. Plant Cell 17,34513469

58 Maeda, H. et al. (2006) Tocopherols play a crucial role in lowtemperature adaptation and phloem loading in Arabidopsis. Plant Cell 18, 2710-2732

59 Weckwerth, W. (2003) Metabolomics in systems biology. Annu. Rev. Plant Biol. 54, 669-689
60 Vidi, P.A. et al. (2007) Plastoglobules: a new address for targeting recombinant proteins in the chloroplast. BMC Biotechnol. 7, 4

61 Froehlich, J.E. et al. (2001) Tomato allene oxide synthase and fatty acid hydroperoxide lyase, two cytochrome P450s involved in oxylipin metabolism, are targeted to different membranes of chloroplast envelope. Plant Physiol. 125, 306-317

62 Hirschberg, J. (2001) Carotenoid biosynthesis in flowering plants. Curr. Opin. Plant Biol. 4, 210-218 